\title{
Gender Determined Flexible Sigmoidoscopy Planning
}

\author{
Manish Chand ${ }^{1, *}$, Ted Andrews ${ }^{2}$ and Guy Nash ${ }^{2}$ \\ ${ }^{I}$ Queen Alexandra Hospital, Southwick Hill Road, Cosham, Portsmouth, PO6 3 LY and ${ }^{2}$ Poole General Hospital, \\ Longfleet Road, Poole, BH15 2JB, UK
}

\begin{abstract}
The most common investigation carried out in UK hospitals to investigate bright red rectal bleeding is a flexible sigmoidoscopy (FS). The role of flexible sigmoidoscopy in colorectal screening has not yet been fully ascertained and colonoscopy remains the investigation of choice for the diagnosis of colonic pathology. However, FS is a simple, quick, sensitive and specific investigation to diagnose and even treat some left sided colorectal lesions. We investigated whether there was any difference between male and female patients undergoing FS in terms of duration and extent of procedure, and describe for the first time a significant increase in time taken to complete the examination in females. As eleven males may be scoped in a similar time to ten females this may have bearing on service efficiency.
\end{abstract}

\section{INTRODUCTION}

Rectal bleeding may be a feature of several disorders within the gastro-intestinal tract (GIT) including cancers. In the UK, such a symptom would necessitate the referral to a specialist for further investigation. Clinicians may choose one or a combination of several tools to investigate such a symptom, much of which depends on the individual and their history.

In recent years, flexible sigmoidoscopy (FS) has proved to be an invaluable tool for assessment of the lower GIT. It has been shown to be able to detect $65-75 \%$ of polyps and $50-65 \%$ of colorectal cancers [1-3]. Although not as sensitive as colonoscopy which is regarded as the "gold standard" investigation for detecting colonic lesions, it does provide an important means of screening a large segment of the population without the logistical and training issues which surround colonoscopy. Many hospitals have been required to schedule additional sessions to cope with the demand of lower GI endoscopy. Efficient use of this service is necessary to reduce delay and cancellations for patients.

We have investigated whether there is any difference in the duration or extent of the procedure between the sexes of the patient to determine if this can help in better planning of FS sessions.

\section{METHOD}

We conducted a prospective study involving all patients undergoing a FS over an 18 month period (April 2005 - October 2006). All patients described the symptom of bright red rectal bleeding which had led to a FS. Patients who were investigated with FS as a primary procedure were those classified as "low risk" for colorectal cancer. Criteria for this included age less than 55; no constitutional symptoms; no clear family history of colorectal cancer; no associated change in bowel habit.

*Address Correspondence to this author at the Queen Alexandra Hospital, Southwick Hill Road, Cosham, Portsmouth, PO6 3LY, UK;

E-mail:mans001@aol.com
Within the defined group exclusion criteria were emergency procedures; patients which required sedation or antispasmodics; patients which had additional associated procedures at the time, eg polypectomy; patients which had undergone previous colorectal or abdomino-pelvic surgery. The FS were carried out by one of four consultant surgeon endoscopists with Pentax flexible fibreoptic sigmoidoscopes (FS34W) - [Pentax Medical Company, Montvale, NJ 07645, USA]. None of the procedures were included from teaching lists and involved only the consultant.

Patients received bowel preparation in the form of two $5 \mathrm{mg}$ BISACODYL (NON PROPRIETARY) tablets taken on $6 \mathrm{pm}, 2$ days before the procedure. The day before the procedure (light diet and a glass of clear fluid every hour) as well as 2 further tablets at 8 am and $6 \mathrm{pm}$. Then on the morning of the procedure, Micolette Micro-enema (Pinewood Laboratories Limited, Ballymacarbry, Ireland) enema [sodium citrate $450 \mathrm{mg}$, sodium lauryl sulphoacetate $45 \mathrm{mg}$, glycerol $625 \mathrm{mg}$ in $5 \mathrm{ml}$ dose with nozzle for rectal administration] 90 minutes before procedure.

The duration of the procedure from intubation of the anal canal to the withdrawing of the endoscope was recorded by nursing staff. This information is routinely recorded for audit purposes by the endoscopy nurses. The extent of the procedure was also recorded in terms of which part of the colon was reached. We defined the end-point of the procedure as the endoscopist reaching what they considered was the splenic flexure.

ANOVA statistical analysis was used to analyse the data according to the sex of the patient.

The data used for this study was routinely collected data from the endoscopy unit. This was discussed and approved with the research and ethics committee.

\section{RESULTS}

A total of 1515 patients were included in the analysis. Of these, 749 were male and 766 were female. Both groups were comparable for age. 
Table 1.

\begin{tabular}{|c|c|c|c|c|}
\hline Male (749) & 13.66 & 12 & 10 & \multirow{2}{*}{0.033} \\
\hline Female (766) & 15.53 & 14 & 10 & \\
\hline
\end{tabular}

All procedures reached the proximal descending colon.

Table 1 shows the time taken to complete FS in both sexes. There is a significant difference in time taken between the sexes with the procedure being quicker in male patients. Statistical significance was accepted as $\mathrm{p}<0.05$. There were no reported significant complications in any of these procedures.

\section{DISCUSSION}

Colonoscopy is now well recognised as the "gold standard" investigation for lesions of the lower GIT [4]. However, difficulties in implementing this procedure on a widespread basis has proved challenging, and furthermore requires greater training and expertise to be performed safely. Deciding which modality of investigation to use can in itself be challenging as different symptoms and age groups imply different pathology. The ideal is to be able to successfully diagnose or exclude colonic pathology in a timely fashion without the need for repeated or further examination. Flexible sigmoidoscopy has allowed a greater number of practitioners to offer a limited colonic investigation and when combined with other tests such as faecal occult blood tests has been shown to be able to detect up to $90 \%$ of malignant lesions [5]. Further, it is thought to be 10-20 times safer in terms of complications than colonoscopy [6].

Several studies have looked at the factors which lead to a difficult or incomplete examination, but this is the first study to look specifically at the time of procedure and related this to efficient service planning. This model is particularly applicable to the UK system where financial and manpower constraints are major factors in healthcare planning but also provide useful information for all endoscopists.

Our results have shown that there is a significant difference in the time taken to perform FS between the sexes. This result may influence the planning of lists which are often predominantly male or female; in our hospital all flexible sigmoidoscopy lists are divided into male or female. Both groups were comparable in their baseline characteristics and our results compares favourably with previous studies highlighting the relative difficulty in endoscopic examinations of the colon in women.

Previous abdominal or pelvic surgery, female sex, age over 65, diverticulosis and laxative use have all been implicated in prolonged or incomplete colonoscopic procedures [7-11]. The majority of such patients were excluded from our study but this information is important when planning lists for service provision or training purposes. One feature which is highly variable between patients is the effectiveness of bowel preparation prior to the procedure. Clearly, if poor or unsatisfactory preparation leads to difficulties in providing an adequate view of the mucosa, one can presume that the procedure will take longer. Whether there is a difference in bowel preparation between the sexes is not known and was not qualitatively analysed in this study.

Another important consideration is that in most units there will be trainee endoscopists in addition to more experienced ones. This will no doubt have an effect on the time taken to complete the procedures safely. FS has a learning curve akin to any skilled procedure however our study only included patients who were examined by gastrointestinal consultants.

It has recently been shown that male patients are more likely to accept FS rather than females [12]. There appears to be complex reasoning behind this, but clearly if a patient is more willing to undergo a procedure beforehand, it is reasonable to presume that they will tolerate it better which may in turn lead to a less difficult examination.

The limitations in this study include not factoring in the adequacy of bowel preparation and accuracy in localising the end-point of the examination. Identifying the site of the sigmoidoscope within the colon is difficult, and localising the descending colon or splenic flexure was subjective for each consultant's experience.

\section{CONCLUSION}

In practical terms, we have found that 11 male patients can be "endoscoped" during a FS session compared with 10 female patients. Using this information, we have divided FS sessions into dedicated male and female lists in order to improve the efficiency of our endoscopy service.

This significant difference in duration of FS between male and female patients may be due to variation in pelvic anatomy, colonic length and laxity of supporting structures within the pelvis. These factors may be replicated in colonoscopy completion times and thus have bearing on surveillance and screening lists.

\section{REFERENCES}

[1] Johnson DA, Gurney MS, Volpe RJ, et al. A prospective study of the prevalence of colonic neoplasms in asymptomatic patients with an age-related risk. Am J Gastroenterol 1990; 85: 969-74.

[2] Lieberman DA, Smith FW. Screening of colonic malignancy with colonoscopy. Am J Gastroenterol 1991; 86: 946-51.

[3] Mueller AD, Sonnenberg A. Prevention of colorectal cancer by flexible sigmoidoscopy and polypectemy: a case control study of 32702 veterans. Ann Intern Med 1995; 123: 904-10.

[4] Atkin W. Implementing screening for colorectal cancer. BMJ 1999; 319: 1212-13.

[5] Sherrick RC. Screening form colorectal cancer. N Engl J Med 2000; 343: 1652-4

[6] Levin TR. Flexible sigmoidoscopy for colorectal screening: valid approach or short-sighted? Gastroenterol Clin North Am 2002; 31: 1015-29. 
[7] Pierzchajlo RP, Ackermann RJ, Vogel RL. Colonoscopy performed by a family physician. A case series of 751 procedures. J Fam Pract 1997; 44: 473-80.

[8] Eloubeidi MA, Wallace MB, Desmond R, et al. Female gender and other factors predictive of a limited screening flexible sigmoidoscopy examination for colorectal cancer. Am J Gastroenterol 2003; 98: 1634-9.

[9] Brill JR, Baumgardner DJ. Establishing proficiency in flexible sigmoidoscopy in a family practice residency program. Fam Med 1997; 27: 580-3.
[10] Anderson JC, Messina CR, Cohn W, et al. Factors predictive of difficult colonoscopy. Gastrointest Endosc 2001; 54: 558-62.

[11] Ramakrishnan K, Sheld D. Predictors of incomplete flexible sigmoidoscopy. J Am Board Fam Pract 2003; 16: 598-602.

[12] Golder S, Weatherly H, Spilsbury K, et al. The effectiveness and cost-effectiveness of respite for caregivers of frail older people. J Am Geriatr Soc 2007; 55(2): 290-9.

Received: November 1, 2008

Revised: January 1, 2009

Accepted: March 9, 2009

(C) Chand et al.; Licensee Bentham Open.

This is an open access article licensed under the terms of the Creative Commons Attribution Non-Commercial License (http://creativecommons.org/licenses/ by-nc/3.0/) which permits unrestricted, non-commercial use, distribution and reproduction in any medium, provided the work is properly cited. 